\title{
Differential sensor in front photopyroelectric technique: I. Theory
}

\author{
R Ivanov ${ }^{1}$, G Gutierrez-Juarez ${ }^{2}$, J L Pichardo-Molina ${ }^{3}$, I Moreno ${ }^{1}$, \\ A Cruz-Orea ${ }^{4}$ and E Marín \\ ${ }^{1}$ Facultad de Física, Universidad Autónoma de Zacatecas, Calz. Solidaridad Esquina Paseo \\ de la Bufa s/n, C.P. 98060, Zacatecas, Zac., México \\ ${ }^{2}$ Instituto de Física, Universidad de Guanajuato, Loma del Bosque 103, Lomas del Campestre, \\ C.P. 37150, León, Gto., México \\ ${ }^{3}$ Centro de Investigaciones en Óptica, Loma del Bosque 115, Lomas del Campestre, C.P. 37150 , \\ León, Gto., México \\ ${ }^{4}$ Departamento de Física, CINVESTAV-IPN, Av. IPN 2508, San Pedro Zacatenco, C.P. 07360, \\ México D.F., México \\ ${ }^{5}$ Centro de Investigación en Ciencia Aplicada y Tecnología Avanzada, Instituto Politécnico Nacional, \\ Legaría 694, Colonia Irrigación, C.P. 11500, México D. F., México \\ E-mail: rumen@planck.reduaz.mx
}

Received 27 December 2007, in final form 16 February 2008

Published 14 March 2008

Online at stacks.iop.org/JPhysD/41/085106

\begin{abstract}
In this paper the theory of the differential front photopyroelectric technique is developed. The thermal effusivity measurements of a sample through photopyroelectric direct (no-differential) experiments do not have sufficient resolution and accuracy to detect small changes in the thermal effusivity. To assess minor variations in this thermal magnitude, differential methods should be used. These methods compare properties of a reference sample and another unknown sample, which are placed separately in both halves of the differential cell. It is shown that in order to achieve better metrological properties of the differential measurement and electromagnetic interference immunity, the signals of both halves must be subtracted directly at the output of the two parallel connected pyroelectric sensors. The thickness of the samples should have the maximum possible value, at least 10 times higher than the thermal diffusion length for minimum frequency. The results of numerical simulations for the amplitude, phase, real and imaginary parts with water as a reference sample and the other sample with a thermal effusivity very close to that of water (contaminated water) are presented. These results show that measurements should be made in the nearly ideal voltage mode, which ensures a better signal-to-noise ratio than the ideal current mode.
\end{abstract}

\section{Introduction}

Photoacoustic (PA) and photothermal techniques (PT) have been used for many years in non-destructive evaluation of different materials. Among the PT, the photopyroelectric (PPE) technique has proved to be a very useful tool for measuring the thermal properties of liquid samples, primarily the thermal effusivity $(e)$ and diffusivity $(\alpha)[1,2]$. The PPE technique has two experimental configurations; the first one is the back photopyroelectric (BPPE) configuration [3-5], in which the sample is illuminated directly. The second is the front photopyroelectric (FPPE) configuration [6-9] in which the PPE detector is illuminated.
From the metrological point of view, each technique can be used in a direct or differential mode. In the direct mode, the physical parameter is measured in an absolute way. In the differential mode, two samples are used, one corresponding to the reference material with well-known parameters, and the other is a sample for which its parameters have been slightly modified. The idea of this mode is to obtain the difference between the parameters. Knowing this difference and the corresponding parameter value of a reference material, we can find the parameter value of the unknown sample with high accuracy. Both types of measurements are very useful; however, when the objective is to measure small variations 


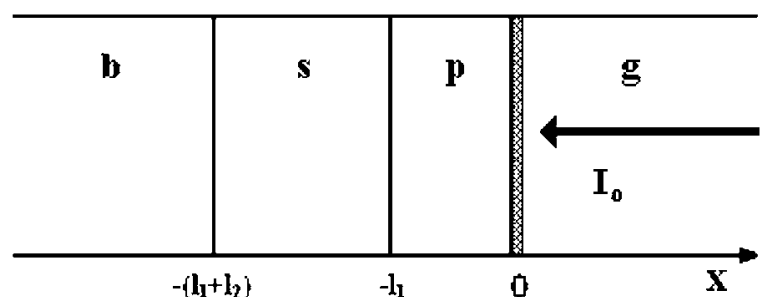

Figure 1. Geometry of the conventional FPPE cell. The sensor has metallized faces for electrical contacts.

of the physical parameters, the direct method does not offer enough resolution to detect small changes and the differential method must be used.

The PPE technique has mainly been used in the direct mode [3-11], restricting the use of the technique to the analysis of pure materials, or materials, with large differences in their thermal properties that must be determined. Samples with very closer thermal parameters can be characterized with PPE differential methods $[12,13]$. These kinds of samples are obtained, for example, when a reference material has been slightly modified (impurities, contaminations, chemical and photochemical reactions, etc).

This work is the first of two papers, and is focused to present and discuss the theory of what we will call the differential FPPE (D-FPPE) technique.

\section{Direct FPPE theory}

In what follows we will introduce the direct (no-differential) FPPE theory with minimal approximations.

\subsection{Direct FPPE temperature field}

A sketch of the conventional FPPE cell is shown in figure 1. Here, $g$ is the air, $p$ is the pyroelectric sensor with thickness $l_{1}$, $s$ is the sample with thickness $l_{2}$ and $b$ is the backing. $I_{0}$ is the intensity of the incident modulated light beam, which impinges onto the pyroelectric sensor at $x=0$.

It has been previously shown [7] that the PPE signal, in voltage or current mode, is proportional to the average temperature along the pyroelectric sensor, which can be calculated by solving the system of heat diffusion equations with properly boundary conditions for the geometry shown in figure 1 . Then, the average temperature for an optically opaque pyroelectric sensor is given by

$$
\left\langle T_{\mathrm{p}}(t, \omega)\right\rangle=\frac{I_{0}(1-R)}{4} \Gamma \exp (\mathrm{i} \omega t)
$$

where

$$
\begin{aligned}
\Gamma= & \frac{\left(1-\mathrm{e}^{-\sigma_{\mathrm{p}} l_{1}}\right)}{\sigma_{\mathrm{p}} l_{1}} \frac{\left(1+R_{\mathrm{gp}}\right)}{k_{\mathrm{p}} \sigma_{\mathrm{p}}} \\
& \times\left[\frac{\left(R_{\mathrm{sp}}+R_{\mathrm{bs}} \mathrm{e}^{-2 \sigma_{\mathrm{s}} l_{2}}\right) \mathrm{e}^{-\sigma_{\mathrm{p}} l_{1}}+\left(1+R_{\mathrm{sp}} R_{\mathrm{bs}} \mathrm{e}^{-2 \sigma_{\mathrm{s}} l_{2}}\right)}{R_{\mathrm{gp}} \mathrm{e}^{-2 \sigma_{\mathrm{p}} l_{1}}\left(R_{\mathrm{sp}}+R_{\mathrm{bs}} \mathrm{e}^{-2 \sigma_{\mathrm{s}} l_{2}}\right)-\left(1+R_{\mathrm{sp}} R_{\mathrm{bs}} \mathrm{e}^{-2 \sigma_{\mathrm{s}} l_{2}}\right)}\right] .
\end{aligned}
$$

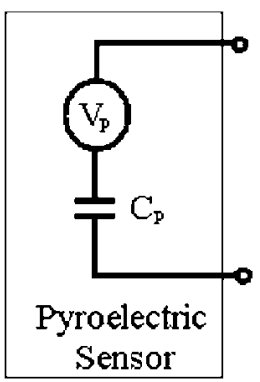

Figure 2. Equivalent diagram of a pyroelectric sensor [14].

In equation (2) the following notation has been used:

$$
\begin{aligned}
& R_{i j}=\frac{1-b_{i j}}{1+b_{i j}}, \quad b_{i j}=\frac{e_{i}}{e_{j}}, \quad \sigma_{j}=(1+i) a_{j}, \\
& a_{j}^{-1}=\mu_{j}=\sqrt{\frac{\alpha_{j}}{\pi f}} .
\end{aligned}
$$

In the above equations $\omega=2 \pi f$ with $f$ as the incident radiation chopping frequency, $\alpha_{i}, e_{i}$ and $k_{i}$ are the thermal diffusivity, effusivity and conductivity of the $i$ th region of figure $1(i=\mathrm{s}, \mathrm{b}, \mathrm{p}), R$ is the fraction of the optical radiation reflected by the front electrode of the sensor, $\mu_{i}$ is the so-called thermal diffusion length and the parameter $R_{i j}$ represent the thermal wave reflection coefficient at the $i-j$ interface.

\subsection{Direct FPPE signal}

It has been shown $[1,3,4]$ that the average pyroelectric voltage is given by

$$
V(t)=\frac{p l_{1}}{\varepsilon}\left\langle T_{\mathrm{p}}(x, t)\right\rangle,
$$

where $p$ and $\varepsilon$ are the pyroelectric coefficient and the permittivity constant of the pyroelectric material, respectively. Substituting equation (1) in equation (3) leads to

$$
\begin{aligned}
V(t) & =\frac{p l_{1} P_{0}(1-R)}{4 \varepsilon A_{\mathrm{p}}} \Gamma \exp (\mathrm{i} \omega t) \\
& =\frac{p P_{0}(1-R)}{4 C_{\mathrm{p}}} \Gamma \exp (\mathrm{i} \omega t),
\end{aligned}
$$

where $A_{\mathrm{p}}$ and $C_{\mathrm{p}}$ are the pyroelectric area and capacitance, respectively. In this equation, it has been assumed that the pyroelectric sensors are impinged by a modulated laser beam of power amplitude $P_{0}$.

In order to obtain the direct FPPE signal we will use the equivalent diagram proposed in [14] which is given in figure 2, where $V_{\mathrm{p}}$ is the equivalent voltage generator, whose value $V(t)$ is in general a complex number. This voltage is often measured using a lock-in amplifier. Measurements can be performed in both, amplitude-phase and in-phase-quadrature channels, the later corresponding to the real and imaginary parts of the signal, respectively. At low frequencies only minimal losses appear. For this reason, the resistances will not be taken into account in the equivalent diagram.

Figure 3 shows the results of theoretical calculations (all simulations in this paper were performed with Mathematica ${ }^{\circledR}$ ) of the amplitude, phase, imaginary part and negative real part 
of $V(t)$ for a thick water sample. The negative real part of $V(t)$ was chosen in order to use a logarithm scale. The parameters used were $l_{1}=28 \mu \mathrm{m}, l_{2}=$ infinity, $R=0, C_{\mathrm{p}}=1 \mathrm{nF}$ and $C_{k}=200 \mathrm{pF}$. The water thermal properties were taken as $\alpha_{\mathrm{w}}=0.145 \times 10^{-6} \mathrm{~m}^{2} \mathrm{~s}^{-1}$ and $e_{\mathrm{w}}=1600 \mathrm{~W} \mathrm{~s}^{1 / 2} \mathrm{~m}^{-2} \mathrm{~K}^{-1}$; air was assumed as backing material $\left(\alpha_{\mathrm{g}}=22.260 \times\right.$ $10^{-6} \mathrm{~m}^{2} \mathrm{~s}^{-1}, e_{\mathrm{g}}=5.510 \mathrm{~W} \mathrm{~s}^{1 / 2} \mathrm{~m}^{-2} \mathrm{~K}^{-1}$ ) [15]. The PVDF thermal properties were taken as $\alpha_{\mathrm{p}}=0.06 \times 10^{-6} \mathrm{~m}^{2} \mathrm{~s}^{-1}$ and $e_{\mathrm{p}}=559.4 \mathrm{~W} \mathrm{~s}^{1 / 2} \mathrm{~m}^{-2} \mathrm{~K}^{-1}$ [14]. These graphs are in agreement with experimental results in the ideal voltage mode.

\section{Differential FPPE sensor theory}

\subsection{Choice of the electrical circuit}

The accuracy of single (non-differential) FPPE cell is limited by the external noises and uncertainties of the experimental setup [16, 17]. The external noises and uncertainties are due to: temperature fluctuations, capacitive pick-up of electromagnetic fields $(50 / 60 \mathrm{~Hz})$, intensity fluctuations and noise of the optical excitation source, optical misalignment,

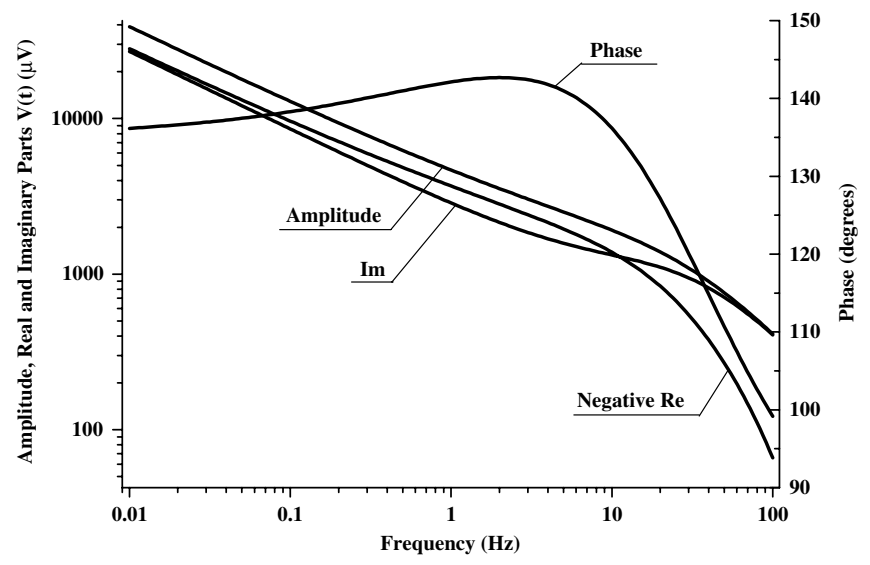

Figure 3. Amplitude, phase, imaginary part and negative real part of direct signal of $V(t)$. The sample used in the simulation was water, and air was chosen as the baking material, as usually. external mechanical noise, preamplifier and lock-in amplifier uncertainties and noises, etc [18]. Because the detection of small thermal effusivity variations by means of amplitude measurements in direct (non-differential) FPPE experiments has become a difficult task some authors [6] have resorted to the measurement of the phase instead of the amplitude of the PPE signal, because in the former channel the relative error of the lock-in becomes small, i.e. lower than $0.01^{\circ}$ [19]. One advantage of this method is that it is relatively insensible to laser power fluctuations. But in order to obtain relative errors lower than $1 \%$ for the thermal effusivity the authors using these method are often themselves forced to repeat several times the same measurement. Unfortunately, as we are going to see later, as differential measurements involved very small values of the relative changes in thermal effusivity, $\Delta e / e$, the signal phase is little sensible to these changes, for that reason one cannot follow this way.

The term 'differential mode' is applied to any sensor where two signals have been subtracted. The diagram of the proposed differential FPPE (D-FPPE) sensor with the measurement electronics is shown in figure 4. This is composed of two identical non-differential FPPE sensors.

According to figure 4, it is possible to subtract signals in points $\mathrm{A}, \mathrm{B}, \mathrm{C}$ or $\mathrm{D}$. Some researchers prefer to do the subtraction of the signals in point $\mathrm{D}$ by means of a computer [12]. However the resolution of each sensor is low, and small changes in the effusivity cannot be detected. If the subtraction of the signals is made in point $\mathrm{C}$ (using $\mathrm{A}-\mathrm{B}$ input of the lock-in amplifier), the uncertainties and noises generated by the preamplifier and by the early steps of the lock-in cannot be avoided. In addition, this input cannot be used to do measurements in the current mode of the lock-in. Moreover, it is possible to saturate the preamplifier and the early stages of the lock-in, if one or both halves of the differential sensor send a large signal. For this reason, high power lasers cannot be used, and that limits the signal-to-noise ratio. Similar consequences are obtained if the subtraction is made in point $B$. Therefore, one can see that the best point to do the subtraction

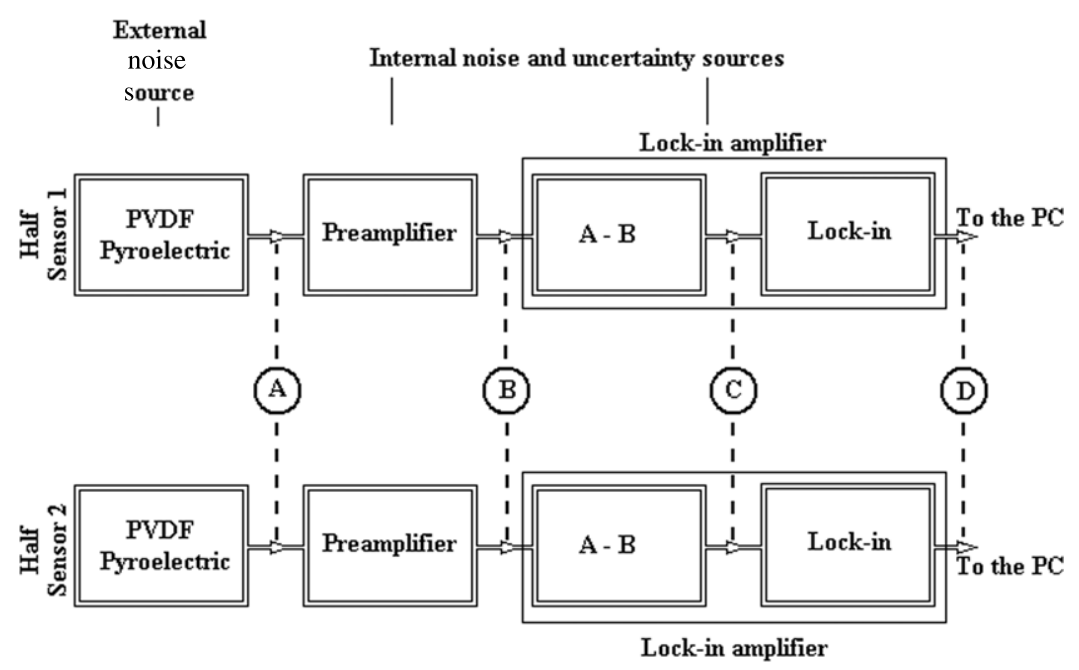

Figure 4. Diagram of the proposed differential FPPE sensor. It is composed of two identical non-differential FPPE sensors. The preamplifier is necessary if it is working close to the ideal voltage mode. 
(a)

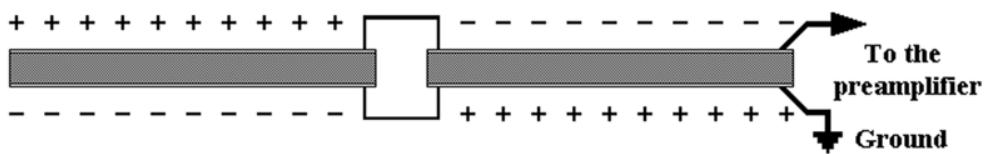

(b)

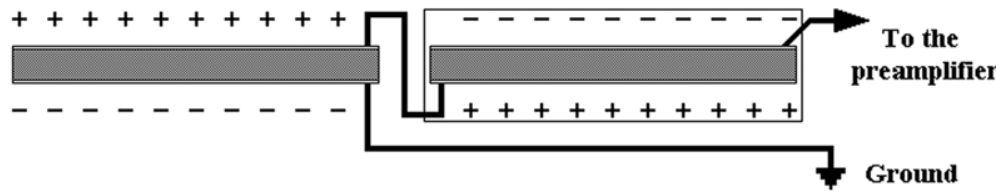

Figure 5. Electrical diagram for the connection between two pyroelectric transducers, $(a)$ in parallel $(b)$ in series. For the in-series connection, the pyroelectric transducer in the square is not grounded.

is A, i.e. subtract signals directly from the pyroelectric transducers.

\subsection{Choosing the type of electrical connection between two pyroelectric transducers}

The electrical connection between two pyroelectric transducers can be done in two ways: in parallel or in series (figure 5).

By using a beam splitter, the laser beam can be divided in two beams with the same power and chopping phase (common mode) so that if the environments of the transducers are equal, they should produce electrical signals with a phase difference equal to $180^{\circ}$ (opposite phase). The illumination in common mode has the advantage that the laser noise produces signals with the same magnitude and sign in pyroelectric transducers. Thus, the subtraction of the signals can reduce the influence of noise and instability of the laser. This does not happen if the illumination is made in the regime of the opposite phase or with two different lasers.

A simulation of electric behaviour for the in-parallel and in-series connection, made with the OrCAD program, shows that if the connections are closer to the ideal current mode the parallel configuration generates a signal twice than that of the in-series connection. However, if the transducers are close to the ideal voltage mode, the signal for the in-series configuration is twice than that of the parallel configuration. On the other hand, in the parallel configuration, both pyroelectric transducers are grounded which decreases the interferences with external electric and magnetic fields $(50 / 60 \mathrm{~Hz}$ electrical line, electric shocks, etc). The in-series configuration always has a pyroelectric transducer with floating potential (in a square in figure 5), which is affected by the external noise sources and makes the mechanical design of the sensor difficult. Additionally, in order to work in a regime closer to the ideal voltage mode the use of a high resistance preamplifier is recommended, while in ideal current mode regime the use of the lock-in amplifier along is sufficient [17]. One drawback of the in-parallel configuration, as compared with the in-series configuration in a differential experiment, is that it is not possible to measure the signal generated only in one of the pyroelectric transducers. After considering the advantages and the disadvantages of each type of connection we have chosen the in-parallel configuration.

As the differential FPPE sensor has been designed in order to measure small differences between thermal properties of two

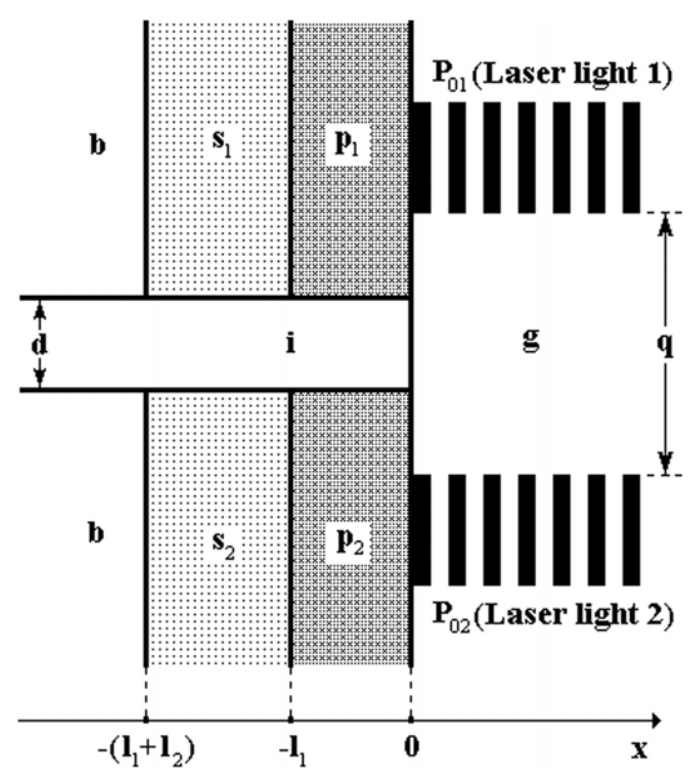

Figure 6. Differential FPPE cell. Here $l_{1}$ and $l_{2}$ are the pyroelectric $(p)$ and sample $(s)$ thicknesses, respectively. $d$ and $q$ are the distances between the two halves of the cell and the laser light beams, respectively. $b$ and $i$ are the backing and thermo-insulator layer, respectively. $P_{01}$ and $P_{02}$ are the powers of the incident modulated light beams impinging onto the pyroelectric sensors at $x=0$.

materials, the desired signal is small and the electronics in the experimental setup can reduce it. So, it is recommended to use measurement systems in the voltage and current modes, which should be as close as possible to the ideal regimes. In the following sections, only the ideal regimes will be discussed.

\section{Theoretical model}

A sketch of the differential FPPE cell is shown in figure 6 . Here, $g$ is the air, $p_{1}$ and $p_{2}$ are the pyroelectric sensors with thickness $l_{1}, s_{1}$ and $s_{2}$ are the sample with thickness $l_{2}, b$ is the backing and $q$ is the distance between both laser beams. Cell halves are separated by a distance $d$ which contains a thermal insulator i. $P_{01}$ and $P_{02}$ are the powers of the incident modulated light beam, which impinges onto the pyroelectric sensor at $x=0$. The 'laser light 1' and 'laser light 2' are modulated in common mode, because they are produced by a beam splitter. 


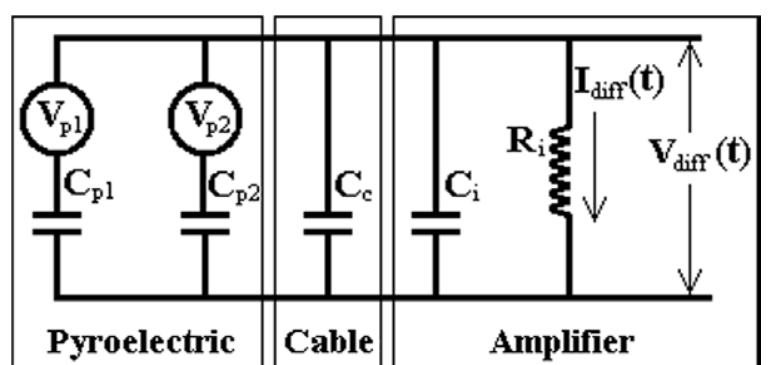

Figure 7. Equivalent diagram for the electrical circuit for the system 'pyroelectric sensor + coaxial cable + amplifiers input'. Details are in the text.

We have performed a digital simulation in order to determine the necessary conditions to implement the approximation of 'thermally thick sample' in the case of a differential sensor. The selected criterion was that the relative difference, $M(\%)$, between the signal amplitude for the finite thickness signal, $V$ (finite), and the amplitude corresponding to the infinite thickness, $V(\infty)$, i.e.

$$
M(\%)=\frac{V(\text { finite })-V(\infty)}{V(\infty)} 100 \%
$$

must be less than $0.1 \%$ for a thermal effusivity relative change from $0.01 \%$ to $10 \%$ for all frequencies. The simulation revealed that the thicknesses of the samples should have a maximum possible value of at least 10 times the thermal diffusion lengths corresponding to the minimum frequency. To avoid an $a c$ heat interchange between samples $s_{1}$ and $s_{2}$, the simulation shows that similar conditions must be fulfilled for the gaps $d$ and $q$ of the figure 6, i.e.

$$
\begin{aligned}
& d>\max \left(10 \mu_{p, \max }, 10 \mu_{i, \max }, 10 \mu_{b, \max }\right), \\
& q>\max \left(10 \mu_{p, \max }, 10 \mu_{s, \max }, 10 \mu_{g, \max }\right),
\end{aligned}
$$

where $\mu_{p, \max }, \mu_{i, \max }, \mu_{b, \max }, \mu_{s, \max }$ and $\mu_{g, \max }$ are the thermal diffusion lengths for minimum frequency for the pyroelectric material, the thermal insulator, the backing material, the sample and air, respectively.

The equivalent diagram for the electrical circuit, in the system 'pyroelectric sensor + coaxial cable + amplifiers input', is given in figure 7 . In this diagram, $V_{p 1}, V_{p 2}, C_{p 1}$ and $C_{p 2}$ are equivalent voltage generators and capacitances of the pyroelectric sensors $p_{1}$ and $p_{2}$, respectively, $C_{\mathrm{c}}$ is the coaxial cable capacitance, $C_{\mathrm{i}}$ and $R_{\mathrm{i}}$ are the capacitance and resistance of the input of the preamplifier or the lock-in amplifier. $V_{\text {diff }}(t)$ and $I_{\text {diff }}(t)$ are the measured voltage and electrical current, respectively.

\subsection{Ideal voltage mode}

The equivalent diagram in the ideal voltage mode is shown in figure 8 , where $C_{k}=C_{\mathrm{c}}+C_{\mathrm{i}}$ is the resultant capacitance of cable and preamplifier input capacitances.

Applying Kirchhoff's laws to the circuit in figure 8 it can be shown that

$$
V_{\mathrm{diff}}(t)=\frac{C_{p 2} V_{p 2}-C_{p 1} V_{p 1}}{C_{p 1}+C_{p 2}+C_{k}}
$$

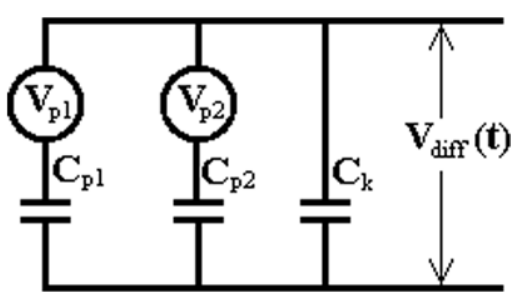

Figure 8. Equivalent diagram in the ideal voltage mode.

Substituting equation (4) in equation (5), we obtain

$$
\begin{aligned}
& V_{\text {diff }}(t)=\frac{p \mathrm{e}^{\mathrm{i} \omega t}}{4\left(C_{p 1}+C_{p 2}+C_{k}\right)} \\
& \quad \times\left[\left(1-R_{2}\right) P_{02} \Gamma_{2}-\left(1-R_{1}\right) P_{01} \Gamma_{1}\right],
\end{aligned}
$$

where $R_{1}$ and $R_{2}$ are the optical reflection coefficients for the respective pyroelectric sensors. As seen from equation (2), if samples $s_{1}$ and $s_{2}$ are the same materials, the coefficients $\Gamma_{1}$ and $\Gamma_{2}$ must be equal; in this case $V_{\text {diff }}(t)$ must always be equal to zero for all frequencies. This is fulfilled if

$$
\left(1-R_{2}\right) P_{02} \equiv\left(1-R_{1}\right) P_{01}=(1-R) P_{0} .
$$

In this case, for two different materials as samples, equation (6) becomes

$$
V_{\text {diff }}(t)=A_{0}\left(\Gamma_{2}-\Gamma_{1}\right) \exp (\mathrm{i} \omega t)
$$

where

$$
A_{0}=\frac{p P_{0}(1-R)}{4\left(C_{p 1}+C_{p 2}+C_{k}\right)} .
$$

Assuming that the thermal properties of the samples are very close, we can write $\alpha_{2}=\alpha_{1}+\Delta \alpha$ and $e_{2}=e_{1}+\Delta e$ and then $\Gamma_{2}=\Gamma_{1}+\Delta \Gamma$. Therefore, equation (8) becomes

$$
V_{\text {diff }}(t)=A_{0} \Delta \Gamma \exp (\mathrm{i} \omega t),
$$

where

$$
\Delta \Gamma=\frac{\partial \Gamma}{\partial \alpha_{\mathrm{s}}} \Delta \alpha_{\mathrm{s}}+\frac{\partial \Gamma}{\partial e_{\mathrm{s}}} \Delta e_{\mathrm{s}}+\eta
$$

where $\eta$ represents the higher order terms and is approximately equal to zero when $\Gamma_{2} \approx \Gamma_{1}$.

For the case of interest of thermally thick samples the parameter $\Gamma$ does not depend on the samples thermal diffusivities, $\alpha_{\mathrm{s}}$ so that the first derivative of the right-hand side of equation (11) becomes equal to zero.

Thus

$$
V_{\text {diff }}(t)=A_{0} \frac{\partial \Gamma}{\partial e_{\mathrm{s}}} \Delta e_{\mathrm{s}} \exp (\mathrm{i} \omega t)
$$

In an actual experiment, determination of $A_{0}$ from the measured values of $p, P_{0}, R, C_{p 1}, C_{p 1}$ and $C_{k}$ is difficult. For this reason, in order to find the $A_{0}$ value, a normalization process with two different liquids which have known thermal parameters must be done. 


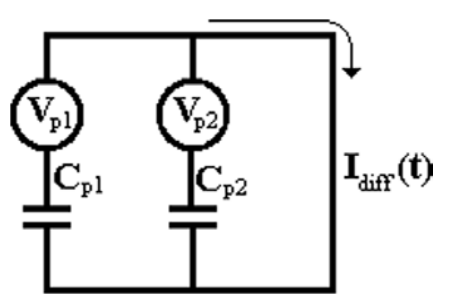

Figure 9. Equivalent diagram in the ideal current mode.

\subsection{Ideal current mode}

Applying Kirchhoff's laws to the circuit represented schematically in figure 9 , it can be shown that

$$
I_{\text {diff }}(t)=\mathrm{i} \omega\left(C_{p 2} V_{p 2}-C_{p 1} V_{p 1}\right) .
$$

Substituting equation (4) in equation (13), we obtain

$$
I_{\text {diff }}(t)=\frac{\mathrm{i} \omega \mathrm{pe}^{\mathrm{i} \omega t}}{4}\left[\left(1-R_{2}\right) P_{02} \Gamma_{2}-\left(1-R_{1}\right) P_{01} \Gamma_{1}\right]
$$

For the ideal current mode, assuming the same conditions as those in the voltage mode, we obtain

$$
I_{\text {diff }}(t)=B_{0} \frac{\partial \Gamma}{\partial e_{\mathrm{s}}} \Delta e_{\mathrm{s}} \exp (\mathrm{i} \omega t)
$$

where

$$
B_{0}=\frac{1}{4} \mathrm{i} \omega p P_{0}(1-R) .
$$

As in ideal voltage mode, in order to find the $B_{0}$ value a normalization procedure with two different liquids of wellknown thermal parameters must be done.

\section{Numerical evaluations and discussion}

As mentioned earlier our analysis will be devoted to thermally thick samples for which we must use only three variables in a numerical simulation, namely $\omega, e$ and $\Delta e$. Other parameters are assumed to be constants and are the same as those used for the simulations presented in figure 3. Figures 10-13 show the results of numerical simulations made for the amplitude, phase, real part and imaginary part, respectively, of the differential voltage given by equation (8), as a function of the modulation frequency. In order to obtain these values, equation (8) was parametrized to $\Delta e / e$. The thermal effusivity was changed by $\Delta e / e=0.003,0.01,0.03$ and 0.10 . In figure 10, the noise level is typical for our experimental setup in the voltage mode, where the main contribution comes from the lock-in amplifier.

As seen in figures 10-13, the amplitude, real part and imaginary part of the differential voltage, equation (8), decrease when the changes in thermal effusivity diminish. The phase is less sensitive to these changes, so it is useless to measure the thermal effusivity changes. Additionally, as seen in the amplitude graph, the noises in the lock-in amplifier reduce the accuracy of the technique, and changes in the thermal effusivity lower than $0.3 \%(\Delta e / e=0.003)$ are difficult to be detected from the theoretical point of view for the assumed laser power $\left(P_{0}=1 \mathrm{~mW}\right)$ and for typical modulation

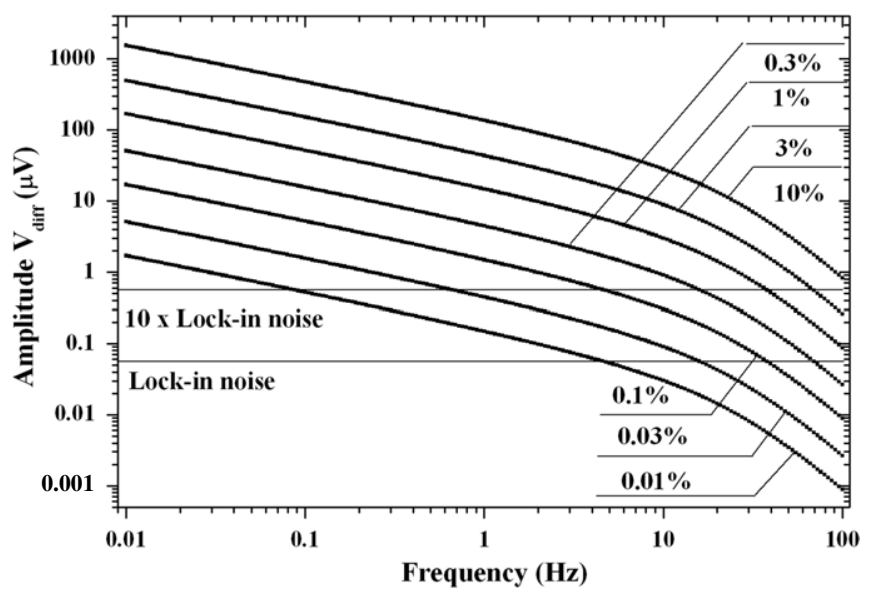

Figure 10. The amplitude of equation (8). Also the noise level multiplied by 10 has been represented as a metrological limit: if the amplitude is smaller than this level, then accurate measurements are impossible.

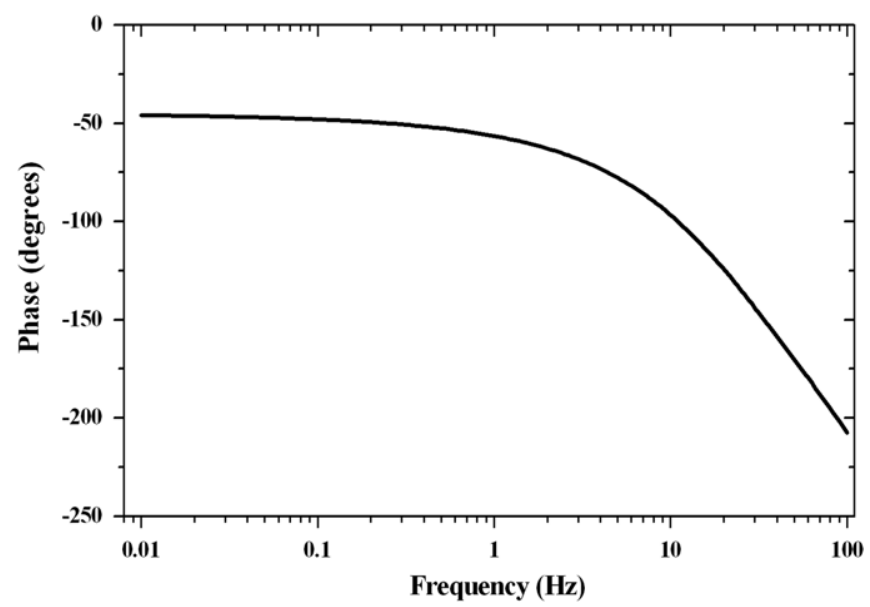

Figure 11. The phase of equation (8). The curves for all values of $\Delta e / e$ are overlapped so we cannot distinguish each of them.

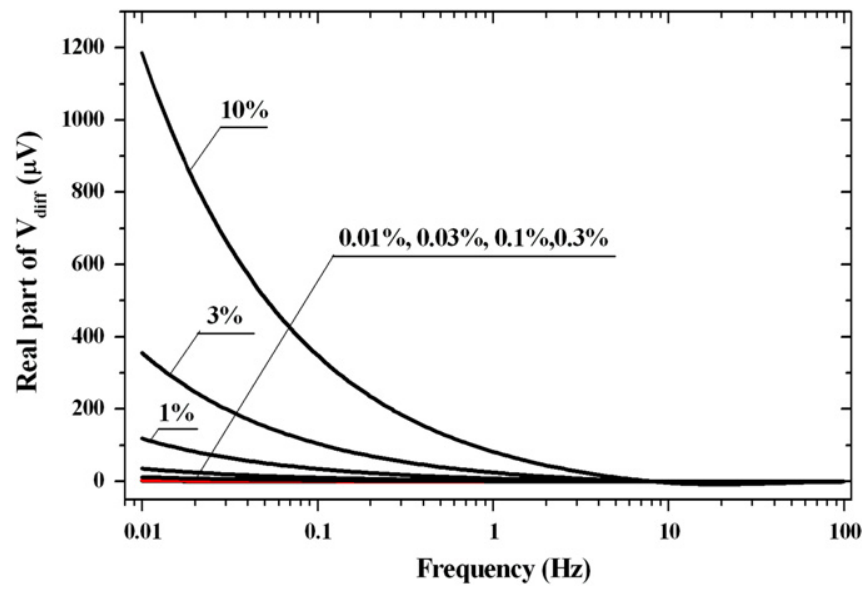

Figure 12. Real part of equation (8).

frequencies $(1-30 \mathrm{~Hz})$. A solution is the use of a more powerful laser.

Figure 14 shows the numerical simulation for the amplitude of the differential current mode, equation (14), as 


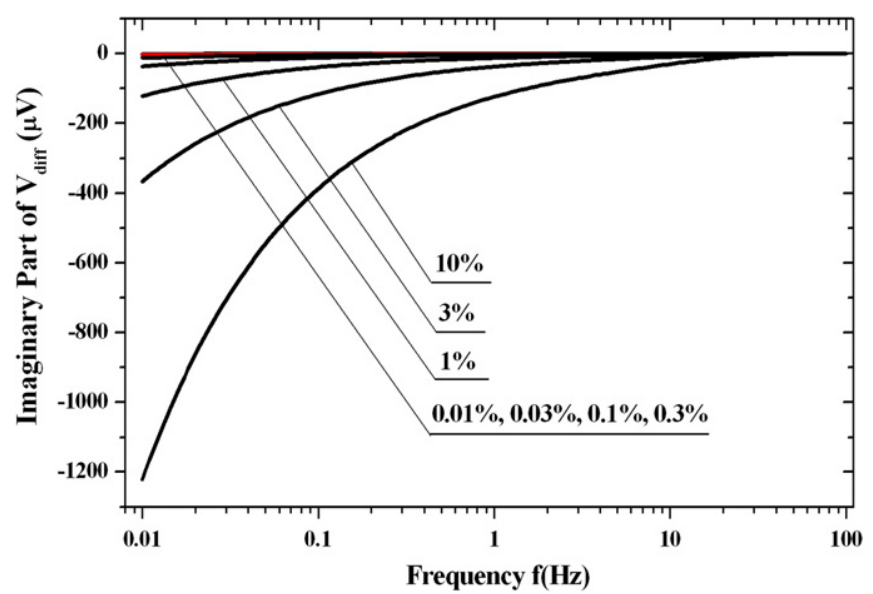

Figure 13. Imaginary part of equation (8).

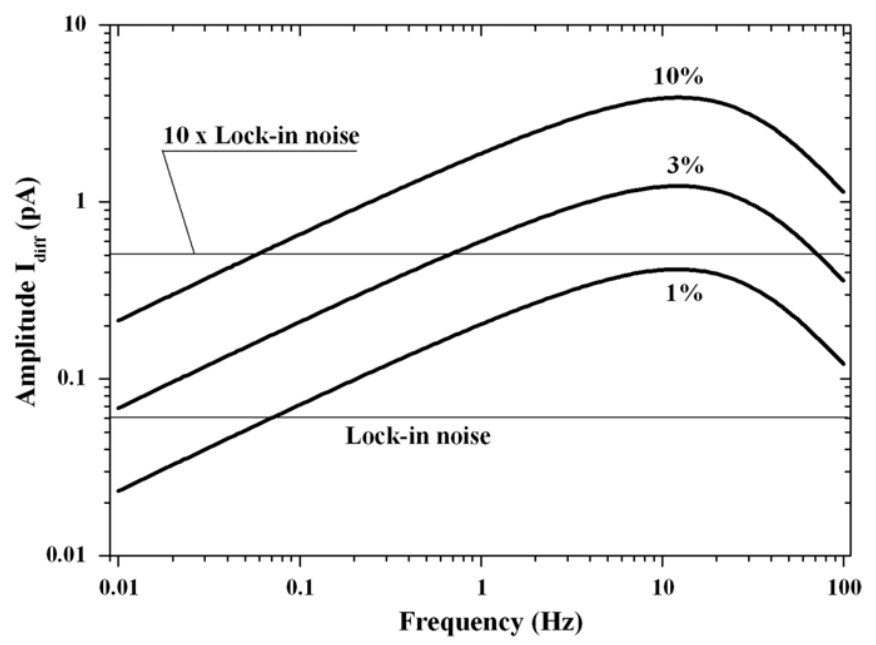

Figure 14. Current mode amplitude. The sample and backing material are water and air, respectively. The parameter values are the same as those used in previous graphs.

a function of the modulating frequency. Again, in order to obtain these values, equation (14) was parametrized to $\Delta e / e$. The sample, backing material and parameters used in this simulation were the same as those used in the simulation of the voltage mode. The thermal effusivity was changed by $\Delta e / e=0.01,0.03$ and 0.10 .

As seen in figure 14, the amplitude values are proportional to $\Delta e$. In the range from 0.01 to $20 \mathrm{~Hz}$, the amplitude increases as a function of the frequency and then rapidly decreases. The different behaviours of the amplitude for the ideal voltage mode and for the ideal current mode occur because the $B_{0}$ coefficient (equation (16)) is directly proportional to the frequency $\omega$. By comparing figures 10 and 14 , it can be seen that the voltage mode is better than the current mode because the signal-tonoise ratio is greater in the first mode for the same measurement conditions. Therefore, it is recommended to work in the regime near the ideal voltage mode. It is also suggested to work with the real and imaginary parts of the differential voltage in order to obtain $\Delta e$ values with more accuracy. This is because the formulae for the real and imaginary parts are simpler than the formula for the amplitude.

\section{Conclusions}

The theory of the differential front photopyroelectric technique was developed. The thermal effusivity measurements of a sample through photopyroelectric direct (no-differential) experiments do not have sufficient resolution and accuracy to detect small changes in the thermal effusivity. In order to assess minor variations in this thermal parameter, differential methods have been proposed. It has been shown that to achieve better metrological properties of the differential measurement, the signals must be subtracted directly between the two pyroelectric sensors connected in parallel. The sample thicknesses should have the maximum possible value, at least 10 times higher than the sample thermal diffusion lengths for the minimum frequency. The results of simulation for the amplitude, phase, real and imaginary part for the water reference sample and samples with thermal effusivity very close to that of water show that measurements should be made in the nearly ideal voltage mode, which ensures a better signalto-noise ratio than the ideal current mode. The subtraction of the signals directly in the pyroelectrics allows the use of more powerful illumination lasers.

\section{Acknowledgments}

This work was financially supported by the Consejo Nacional de Ciencia y Tecnología, Mexico, under grants Nos 67823 and SEP-2003-C02-44058. The authors would like to thank Maureen Sophia Harkins for proofreading this manuscript (cetet@uaz.edu.mx), COFAA and the support received by SIP Project No 20070490, both from IPN.

\section{References}

[1] Chirtoc M, Dadarlat D, Bicanic D, Antoniow J S and Egee M 1997 Progress in Photothermal and Photoacoustic Science and Technology III ed A Mandelis and P Hess (Bellingham, WA: SPIE) p 185

[2] Almond D and Patel P 1996 Photothermal Science and Techniques (London, UK: Chapman and Hall)

[3] Mandelis A and Zver M M 1985 J. Appl. Phys. 574421

[4] Chirtoc M and Mihilescu G 1989 Phys. Rev. B 409606

[5] Delenclos S, Chirtoc M, Sahraoui A H, Kolinsky C and Buisine J M 2002 Rev. Sci. Instrum. 732773

[6] Dadarlat D and Neamtu C 2006 Meas. Sci. Technol. 173250

[7] Dadarlat D and Frandas A 1993 Appl. Phys. A 56235

[8] Dadarlat D, Chirtoc M, Nematu C, Candea R M and Bicanic D 1990 Phys. Status Solidi a 121 K231

[9] Caerels J, Glorieux C and Thoen J 1998 Rev. Sci. Instrum. 692452

[10] Dadarlat D, Bicanic D, Visser H, Mercuri F and Frandas A 1995 JAOCS 72273

[11] Cruz-Orea A, Benefour E H, Jamee P, Chirtoc M, Glorieux C, Pitsi G and Thoen J 2003 Rev. Sci. Instrum. 74818

[12] Christofides C, Ghandi K and Mandelis A 1990 Meas. Sci. Technol. 11363

[13] Pittois S, Van Roie B, Glorieux C and Thoen J 2004 J. Chem. Phys. 1211866

[14] http://www.meas-spec.com Piezo Film Sensors-Technical Manual 
[15] http://physics.nist.gov/PhysRefData/contents.html USA National Institute of Standards and Technology

[16] Gutiérrez-Juárez G, Ivanov R, Pichardo-Molina J L,

Vargas-Luna M, Alvarado-Gil J J and Camacho A 2007 Int J. Thermophys. at press, doi: 10.1007/s10765-007-0261-4
[17] Ivanov R, Gutierrez-Juarez G, Pichardo-Molina J L, Moreno I and Vargas M 2005 Superficies Vacio 1817 (in Spanish)

[18] Wang C H and Mandelis A 2000 Rev. Sci. Instrum. 711961

[19] http://www.thinksrs.com/downloads/PDFs/ Manuals/SR830m.pdf 